\title{
An attempt to estimate the minimal number of Poles infected and treated for malaria in Poland and abroad
}

\author{
Anna Kuna ${ }^{1,2}$, Beata Szostakowska ${ }^{2}$, Wacław L. Nahorski ${ }^{3}$, Małgorzata Stępień ${ }^{4}$, \\ Danuta Kowalczyk ${ }^{5}$, Joanna Stańczak², Przemysław Myjak $^{2}$
}

\begin{abstract}
${ }^{1}$ Department of Tropical and Parasitic Diseases, University Centre for Maritime and Tropical Medicine, Gdynia, Poland ${ }^{2}$ Department of Tropical Parasitology, Institute of Maritime and Tropical Medicine in Gdynia, Medical University of Gdansk, Poland ${ }^{3}$ Department of Tropical and Parasitic Diseases, Institute of Maritime and Tropical Medicine in Gdynia, Medical University of Gdansk, Poland ${ }^{4}$ Department of Epidemiology, National Institute of Public Health - National Institute of Hygiene, Warsaw, Poland ${ }^{5}$ Outpatients Clinic for Occupational Medicine and Tropical Diseases, University Centre for Maritime and Tropical Medicine, Gdynia, Poland
\end{abstract}

\begin{abstract}
Background: Malaria is one of the three most dangerous infectious diseases in the world. According to official statistics, there are a few dozen cases in Poland annually while the number of Poles treated abroad or self-treating remains unknown. Poland has been declared to be malaria-free since 1963 and nowadays all cases are imported. The aim of the study is to determine the minimal number of malaria cases in Poles at home and abroad in the last decade.

Materials and methods: The medical records of 4,710 patients tested for malaria in the Department of Tropical Parasitology in the years 2003-2012 were analysed. Two spreadsheets were created, which only included people with a history of malaria diagnosed in the reference centre where indirect immunofluorescent-antibody assay (IFA) for Plasmodium falciparum antigen proved positive. The minimum number of Poles who have had malaria at home and abroad was calculated on the basis of positive IFA results; the rate of all treated malaria patients in Poland in relation to those treated in the reference centre and the actual number of Poles with malaria diagnosed at home was calculated.

Results: A group of 376 people with positive serologic tests results in indirect immunofluorescent antibody assay with titre $\geq 1: 20$ were received, including 227 patients with positive serologic results with titre $\geq 1: 80$. The rate of the overall number of malaria cases in Poland compared to the number of malaria cases in the University Centre for Maritime and Tropical Medicine Hospital was determined as 3.47:1. It was demonstrated that every year at least 174 to 211 Poles staying abroad may suffer from malaria. Conclusions: This is the first attempt to estimate the minimal number of Poles infected and treated for malaria in Poland and abroad. The estimated number is $8-10$ times greater than the number of registered cases in Poland.
\end{abstract}

(Int Marit Health 2015; 66, 4: 233-237)

Key words: malaria, seroepidemiologic studies

\section{INTRODUCTION}

Along with HIV/AIDS and tuberculosis, malaria is one of the three most dangerous infectious diseases in the world. Two hundred nineteen million malaria patients were registered in 2010, of which 660 thousand, mainly African children, died. The disease is endemic in
104 countries and territories around the world while the transmission of malaria is present in 97 of them [1]. In Europe, 5,482 cases of malaria were diagnosed in 2011. The confirmed case rate of malaria reported by European Union/European Economic Area countries remains stable, fluctuating around 1 per 100,000 people per year. 
Most cases were imported from African countries (more than 80\%) [2].

In Poland, between a dozen and several dozen patients, including foreigners, suffer from malaria every year. Altogether 92 cases were registered in the years 2009-2012, including 21 cases in 2012, 14 in 2011, 35 in 2010 and 22 in 2009 [3]. Poland has officially been declared free from domestic malaria since 1963, with local transmission persisting until 1957 [4]. Only imported cases are currently registered. It is impossible to determine how many Poles visit tropical countries, especially malaria endemic zones due to lack of research data. Also, the number of Polish citizens with malaria abroad remains unknown as no research has been conducted concerning this problem.

It seems that the approximate number of cases may be determined based on medical history and positive results of serologic tests for malaria as past infection leaves traces in the form of IgG antibodies persisting 8-12 months or longer [5]. An increasing number of malaria cases are being observed not only in travellers but also in missionaries, humanitarian volunteers and professionals working in tropical areas. Only isolated cases of malaria in the visiting friends and relatives (VFRs) group are currently reported in Poland. It is relatively common, especially among missionaries, to undergo self-treatment with antimalarial drugs when the disease is suspected. This may also contribute to difficulties in determining the exact number of Polish malaria patients and results in the fact that the disease is underreported.

The aim of the study is to determine retrospectively the minimal number of malaria cases in Poles at home and abroad in the last decade.

\section{MATERIALS AND METHODS}

The source material consisted of the serologic tests results for parasitic diseases including malaria performed between 2003 and 2012 in the Department of Tropical Parasitology of the Medical University of Gdansk. Indications for serologic testing for malaria included: recurring fever after return from tropical areas, return from malaria-endemic areas, reporting of unusual symptoms and anamnesis indicating malaria in the past [5]. Moreover, microscopic tests (thin and thick blood film) were routinely performed for diagnostic purposes, complemented by immunochromatography [6] and molecular tests [7] in justified cases. Biological samples for diagnostic tests were delivered from the University Centre for Maritime and Tropical Medicine (UCMMiT) Hospital, which includes, among others, the Department of Tropical and Parasitic Diseases and the Outpatients Clinic for Occupational Medicine and Tropical Diseases. These units form, together with the Department of Tropical Parasitology, the National Centre for Tropical Medicine, a tertiary reference centre for diagnosis and treatment of tropical diseases (including malaria) in Poland.
Following the registration of the test results in the Department of Tropical Parasitology, an Excel spreadsheet was created where the data of all patients tested for malaria in the last decade was collected, excluding:

1. Patients tested repeatedly, even if the interval between tests amounted to several years (due to long persistence of antibodies). Only the earliest record was included.

2. Patients with an ongoing malaria infection confirmed by laboratory tests (microscopic, immunochromatographic, molecular).

3. Patients whose samples were delivered from other centres.

4. Foreign nationals. The analysis only concerned Poles. Two derived spreadsheets were subsequently created, which only included people with a history of malaria diagnosed in the reference centre where indirect immunofluorescent-antibody assay (IFA) for Plasmodium falciparum antigen (BioMérieux) proved positive: 1 ) with titre of $\geq 1: 20$ which is considered as diagnostic for malaria, and 2) with titre of $\geq 1: 80$ which is considered as specific for malaria [5].

The minimum number of Poles who have had malaria at home and abroad was calculated on the basis of positive IFA results with titre $\geq 1: 20$, and separately with titre $\geq 1: 80$; the rate of all treated malaria patients in Poland in relation to those treated in the reference centre and the actual number of Poles with malaria diagnosed at home was calculated according to formulae designed by us:

1. Based on IFA titre $\geq 1: 20$

$\chi_{\geq 1: 20}=\frac{\left(a_{\geq 1: 20}+b_{\geq 1: 20}\right) c+d}{10}$

IFA titre $\geq 1: 20$ indicates that the majority of patients with such results have indeed suffered from malaria, although it may have been caused by a non-specific reaction in some of the cases [5].

2. Based on IFA titre $\geq 1: 80$, respectively:

$\chi_{\geq 1: 80}=\frac{\left(a_{\geq 1: 80}+b_{\geq 1: 80}\right) c+d}{10}$

IFA titre $\geq 1: 80$ indicates that all of the persons with such a result have had malaria because it is specific for malaria according to our previous study [5]; where:

- $a_{\geq 1: 20}=$ number of tested serum samples obtained in the reference centre from individuals who were likely to have contracted malaria (IFA positive with titre $\geq 1: 20$ );

- $a_{\geq 1: 80}=$ number of tested serum samples obtained in the reference centre from individuals who have had malaria (IFA positive with titre $\geq 1: 80$ ); 
Table 1. Comprehensive summary of numeric data concerning Poles diagnosed for malaria in the years 2003-2012

\begin{tabular}{|c|c|c|c|c|}
\hline No. & Group of patients (serum samples) & $\mathbf{N}$ & $\%$ & Index \\
\hline 1 & $\begin{array}{l}\text { Number of examined patients, referred for serologic testing for malaria from UCMMiT } \\
\text { and other centres in Poland }\end{array}$ & 4,710 & & \\
\hline 2 & $\begin{array}{l}\text { Number of records (patients, serum samples) with positive IFA results (some patients } \\
\text { were tested multiple times) }\end{array}$ & 712 & & \\
\hline 3 & Number of records (patients) with positive IFA results (each person was recorded only once) & 485 & & \\
\hline 4 & Number of patients with positive IFA results after excluding patients with ongoing malaria & 422 & & \\
\hline 5 & Number of patients from UCMMiT only, with positive IFA results 1:20 and more & 376 & & \\
\hline 6 & Number of patients from UCMMiT only, with positive IFA results 1:80 and more & 227 & & \\
\hline 7 & Number of Poles with malaria in the years 2003-2012 & 222 & & \\
\hline 8 & Number of Polish patients treated for malaria in UCMMiT in the years 2003-2012 & 64 & 28.8 & \\
\hline 9 & Ratio of the number of Poles with malaria in Poland to the number of Poles with malaria in UCMMiT & & & 3.47 \\
\hline
\end{tabular}

$-b_{\geq 1: 20}=$ number of patients diagnosed in the reference centre with a probable medical history of malaria whose IFA result was negative after their return to Poland (estimated according to our previous study);

$-b_{\geq 1: 80}=$ number of patients diagnosed in the reference centre, with a probable medical history of malaria, whose IFA result was negative or low-positive (1:20-1:40) after their return to Poland;

- c $=$ rate of all diagnosed and treated cases of malaria in Poland in relation to the number of malaria cases treated in the UCMMiT Hospital;

- $\quad d=$ actual number of confirmed malaria cases in Poles (the sum of cases registered in the surveillance system and of the known and confirmed cases not registered in the system) in the last decade in Poland;

$-10=$ number of analysed years.

\section{RESULTS}

Altogether, 4,710 serologic tests for anti-Plasmodium spp. antibodies were performed in the analysed 10-year period (2003-2012), of which IFA was positive in 712 cases. After excluding patients in accordance with the aforementioned criteria, ultimately a group of 376 individuals was obtained without ongoing malaria whose IFA was positive with titre $\geq 1: 20$, including 227 persons with IFA-positive results with titre $\geq 1: 80$.

Our earlier study [5] demonstrated that $69 \%$ of IFA tests in patients with laboratory-confirmed malaria contracted in the tropics were reactive with titre $\geq 1: 20$ and $52 \%$ with titre $\geq 1: 80$, while $31 \%$ and $48 \%$ were negative, respectively. Comparison of these earlier results against the present study shows that 376 patients constitute $69 \%$. Therefore, the remaining $31 \%$ is 169 cases (despite confirmation of malaria), which altogether equals $\left(a_{\geq 1: 20}+b_{\geq 1: 20}\right) 545$ cases. In parallel, $52 \%$ of samples were positive in IFA with titre $\geq 1: 80$ and $48 \%$ were negative, which translates into 227 and 209 individuals, respectively (436 altogether).

Based on the data collected from the National Institute of Public Health - National Institute of Hygiene [3] as well as the data from the Department of Tropical Parasitology (the National Centre for Tropical Medicine), it was demonstrated that at least 222 Poles had malaria in the past 10 years, $28.8 \%$ of whom were treated in our centre, and that the ratio of the number of all treated malaria patients in Poland in relation to the number of patients with malaria treated in our centre is 3.47:1. According to our data, it was also revealed that other medical centres failed to report at least 42 cases of laboratory - confirmed malaria in Poles and 6 cases in foreigners to the official surveillance system. All numerical data is presented in Table 1.

By substituting the symbols in equations for numbers we obtain:

$\chi_{\geq 1: 20}=\frac{(376+169) 3.47+222}{10} \approx 211$
$\chi_{\geq 1: 80}=\frac{(227+209) 3.47+222}{10} \approx 174$

This shows that the annual minimal number of Poles with malaria is approximately 174 when taking into account serologic tests results with titre $\geq 1: 80$, and up to 211 when taking into account results with titre $\geq 1: 20$.

\section{DISCUSSION}

The estimated number is 8 to 10 times more than the number of officially registered cases of imported malaria, i.e. acquired abroad and diagnosed in Poland after presentation of symptoms. This number is probably higher because some 
of the analysed patients had malaria more than once, not all of the patients who developed malaria abroad present themselves for follow-up at home and some of the patients who suspect malaria do not consult a physician and self-treat. Only cases of imported malaria are required to be registered in Poland while cases diagnosed and treated abroad should be reported in the country where the infection occurred. Despite a legal obligation to report cases of diseases as listed under regulation [8], some (unknown) number of cases is not included in the statistics because they are underreported by attending physicians. On the other hand, the knowledge of the total annual number of Polish citizens suffering from malaria would make it possible to estimate the real needs of pre-travel counselling and the use of chemoprophylaxis. The knowledge of the total annual number of people at risk of malaria i.e. traveling to endemic areas and of the data concerning the use of chemoprophylaxis among them is crucial for determining the real malaria prevalence among Polish citizens. No system for registration of Poles infected with and treated for malaria abroad exists because neither domestic nor foreign travel agents have such obligation.

The annual number of patients diagnosed with and treated for malaria in Poland registered in the surveillance system is more or less constant and does not exceed several dozen patients a year. Thus, malaria is not a common disease and, therefore, few general practitioners or physicians working at emergency hospital departments include it in differential diagnosis. Although it now seems that the awareness of the risk is gradually increasing due to various educational programmes.

In spite of numerous training events for physicians and awareness-raising campaigns, deaths caused by malaria are still registered and the mortality rate is $7-16$ times higher than in other European countries [4]. It is possible that some deaths are from undiagnosed malaria. Difficulties in the diagnosis of malaria arise not only because of lack of experience amongst physicians, but also because of the atypical course of the disease. The incubation period of malaria plasmodia is prolonged in many cases, often due to the modifying impact of incomplete chemoprophylaxis or due to the influence of other medication e.g. antibiotics taken for other reasons. Therefore, it is not known when to include malaria in differential diagnosis of symptoms after the patient's return from tropical areas. In cases of the most dangerous falciparum malaria, the symptoms occur up to 3 months after being infected, although there is some data describing cases where the symptoms occur after a much longer period [9]. In 2007, a female patient was described to have developed malaria 8 years after her sojourn in the tropics (Angola) [10].

In Poland, the number of immigrants coming from malaria-endemic countries is not significant, therefore, the problem of travelling VFRs does not exist, unlike in other European countries. In the United Kingdom, it is estimated that this group accounted for $40 \%$ of the 2 million travellers to Africa in the year 2000. Yet at the same time VFRs consider themselves resistant to malaria, do not seek medical advice, go on longer trips, often in endemic territories, use prophylaxis treatments reluctantly or are limited financially [11]. A research study conducted in Spain showed that out of 60 malaria cases seen in children between 1995 and 2007, 14 were VFRs, and none applied proper malaria prophylaxis. In addition, the parasitaemia was considerably higher amongst these patients [12].

In Poland, another problem exists - the reluctance of travellers to use malaria prophylaxis, a common belief in the harmfulness of the prophylactic medicines and sometimes a complete lack of knowledge of the existence of malaria in the destination country. Another problem is people's ignorance about the nature of the disease, and their belief in old-fashioned ways of coping with the problem of mosquitoes. It is estimated that approximately $3 / 4$ of Polish patients did not use any prophylaxis against developing malaria (our own study). In connection with an insufficient familiarity with this disease amongst doctors, all of these factors result in a delayed diagnosis and treatment of malaria which endangers infected people's lives.

Since 1963 there were no domestic malaria cases reported in Poland, although there are conditions which might encourage that spread of the disease, namely "malaric" mosquitoes: Anopheles messeae/daciae and An. maculipennis [13]. According to World Health Organisation guidelines, the territory of Poland is stated to be vulnerable, which means that it has the necessary conditions for malaria transmission. Regions prone to malaria transmission should be protected, in particular, against the spread of infected mosquitoes, and influx of people who are already ill or who are suspected carriers of the disease [14]. Patients suffering from malaria arriving in a non-endemic region, other than the danger connected with an unfortunate course of the disease, may contribute to an increase in the risk of transmission as well as the reintroduction of malaria in regions with appropriate climatic conditions and vectors of the disease, as well as play a role in infecting other people through blood transfusions, organ transplants, occupational exposure, and vertical route.

\section{CONCLUSIONS}

According to our calculations, at least 174-211 individuals suffer from malaria, both in Poland and in the tropics, which are 8-10 times more Poles than the number of officially registered imported malaria cases. Moreover, around $20 \%$ of the cases are not registered at all, for a variety of reasons. The task of the epidemiological service is 
to estimate the exact number of people potentially suffering from malaria in Poland, to evaluate the risk of an upward trend in this number and to plan possible actions to combat mosquito breeding sites. In order to limit the risk of malaria transmission it is essential to continue awareness-raising campaigns on chemoprophylaxis as well as other methods which decrease the risk of contracting malaria.

\section{ACKNOWLEDGEMENTS}

The authors would like to express their deepest gratitude to Professor Przemysław Myjak, PhD, for his invaluable support and advice throughout the creation of this article.

\section{COMPETING INTERESTS}

The authors declare that they have no competing interests.

\section{REFERENCES}

1. World Health Organization. World Malaria Report 2013. WHO, Geneva 2013; pp. 31-32.

2. European Centre for Disease Prevention and Control. Emerging and vector-borne diseases: Malaria. In: Annual Epidemiological Report 2013. Reporting on 2011 surveillance data and 2012 epidemic intelligence data. European Centre for Disease Control and Prevention, Stockholm 2013; pp. 131-133.

3. Stępień M, Rosińska M. Imported Malaria in Poland 2003 to 2011: Implications of Different Travel Patterns. J Travel Med 2014; 21: 189-194. doi: 10.1111/jtm.12109.

4. Knap J, Myjak P. Malaria w Polsce i na świecie - wczoraj i dziś. [in Polish] Alfa Medica Press, Bielsko-Biała 2009, p. 67.
5. Myjak P, Jaremin B, Zwierz C et al. Przydatność odczynu immunofluorescencji pośredniej z antygenem Plasmodium falciparum dla bieżącego i wstecznego rozpoznawania malarii. [in Polish] Pol Tyg Lek 1993; 34-36: 732-735.

6. Myjak P, Nahorski W, Żarnowska-Prymek H, Pietkiewicz H. Usefulness of the "OptiMAL Rapid Malaria test” for rapid detection of malaria imported to Poland. Wiad Parazytol 2004; 50: 193-199.

7. Myjak P, Nahorski W, Pieniążek NJ, Pietkiewicz H: Usefulness of PCR for diagnosis of imported malaria in Poland. Eur J Clin Microbiol Infect Dis 2002; 21: 215-218. doi: 10.1007/s10096-001-0690-0.

8. Regulation of the Minister of Health on reporting of diagnosis or suspicion of an infection, infectious disease or death caused by an infection or infectious disease. [in Polish] J Laws Republic Poland, 2013, Item 848.

9. Schwartz E, Parise M, Kozarsky P. Delayed onset of malaria: implications for chemoprophylaxis in travelers. N Engl J Med 2003; 349: 1510-1516. doi: 10.1056/NEJMoa021592.

10. Szmitko P, Kohn ML, Simor AE. Plasmodium falciparum malaria occurring 8 years after leaving an endemic area. Diagn Microbiol Infect Dis 2009; 63: 105-107. doi: 10.1016/j.diagmicrobio.2008.08.017.

11. Bacaner N, Stauffer B, Boulware DR. Travel medicine considerations for North American immigrants visiting friends and relatives. JAMA 2004; 291: 2856-2864. doi: 10.1001/jama.291.23.2856.

12. Arnáez J, Roa M, Albert L. Imported malaria in children: A Comparative Study Between Recent Immigrants and Immigrant Travelers (VFRs). J Travel Med 2010; 17: 221-227. doi: 10.1111/j. 1708-8305.2010.00416.x.

13. Kubica-Biernat B, Kowalska-Ulczyńska B: Identification and distribution of sibling species of Anopheles macullipennis complex (Diptera:Culicidae) in north-eastern Poland. Fragmenta Faunistica; 2011; 54: 17-27. doi: 10.3161/00159301FF2011.54.1.017.

14. Dzbeński T. Epidemiological situation of malaria in Poland: past, present and future. Wiad Parazytol 2008; 54: 205-221. 